The link between ET and PD has frequently been debated in the literature and such patients developing PD after ET are named ETPD, however, the development of dementia has not been described in patients with ETPD. In this study, we have described a clinical case series of 17 patients with initially diagnosed as ET, who developed parkinsonism and subsequent development of dementia. We named this combination of ET, PD and dementia as ETPDD and in these patients, using clinical presentation and FDG-PET studies, the similarities and differences to Parkinson Disease Dementia and other dementia are described. Methods: All patients underwent a detailed neurologic history and examination by same movement disorders specialist. Medical informations of patients were obtained from the patients and family members to clarify the relationship between these disorders. The patient's global cognitive status and severity of dementia were assessed with MMSE and CDR. Demographic and historical data including age, sex, age at tremor onset, duration of postural tremor, type of tremor, age at parkinsonism and dementia onset, the time between ET and PD and PD and dementia, family history of PD, ET, other movement disorders and dementia, and similarities and differences to other dementia were recorded. The diagnosis of PD was established according to the UK-PD Society Brain Bank criteria and the criteria for dementia from DSM-IV was applied. Results: RESULTS Patients who met the following criteria were classified as having ETPDD. (1) presence of both postural-action hand tremor as first symptom on their medical history. (2) symptoms of postural-action tremor for at least 3 to 5 years prior to the onset of other signs of PD.(3) cognitive dysfunctions, impaired activities of daily living and measured by MMSE. Conclusions: Present study specifically focused on the similarities and differences to PD dementia and other dementia, including clinical presentation and FDG-PET studies, leading clinicians to suspect a link among them. To our knowledge, this is the first case series to describe the coincidental occurrence of these three disorders in a single patient.

\section{P4-361 THE INFLUENCE OF MILD COGNITIVE IMPAIRMENT ON MEASURES OF FALL RISK IN COMMUNITY-DWELLING OLDER ADULTS}

Jennifer Blackwood ${ }^{1}$, Tiffany Shubert ${ }^{2}$, Carla Chase $^{3}$, Kieran Fogarty ${ }^{3}$, ${ }^{1}$ University of Michigan-Flint, Flint, Michigan, United States; ${ }^{2}$ University of North Carolina, Chapel Hill, North Carolina, United States; ${ }^{3}$ Western Michigan University, Kalamazoo, Michigan, United States.

Contact e-mail: jblackwo@umflint.edu

Background: Fall related injuries are the fifth leading cause of death in older adults and result in medical costs of more than $\$ 20$ billion per year. Cognitive impairment is a risk for falls and those with mild cognitive impairment (MCI) have changes in gait and are at a greater risk for falling. The purpose of this study was to determine how the presence of MCI as measured with the Montreal Cognitive Assessment tool (MoCA) influences performance on fall risk screening tools: the Five Times Sit to Stand test (FTSTS), usual gait speed, the Timed Up and Go test (TUG) and the Activities Specific Balance Confidence scale (ABC) measures. Methods: Forty seven community dwelling older adults participated. The MoCA was used to stratify the subjects into those with MCI and those without MCI based on a cutoff score of 26. Descriptive statistics and mean scores on each of the fall risk screening tools were reported. Correlations and linear regression analyses were completed. Results: Mean scores on the fall risk tools did not differ between groups. Significant associations between each of the fall risk screening tools were found in those with MCI with the exception of the ABC and gait speed measurements. Regression models were able to predict the TUG and ABC in those with MCI while controlling for age, having a history of falling and taking greater than 4 medications Conclusions: The influence of deficits in cognitive processes reported in those with MCI may influence the interpretation of fall risk measures and the association of those cognitive deficits on fall risk should be considered. Although there were no significant differences detected in overall mean scores, these findings may provide evidence to the subtle influence that early cognitive loss as found in MCI has over overall performance on measures of fall risk. Further studies are needed to examine this influence.

\section{P4-362 FLUCTUATIONS IN OBSTRUCTIVE SLEEP APNEA SEVERITY AND COGNITIVE PERFORMANCE IN MILD ALZHEIMER'S DISEASE}

Kristin Hannesdottir ${ }^{1}$, Solveig Davidsdottir ${ }^{1}$, Arndis Valgardsdottir ${ }^{1}$, Erna Arnardottir ${ }^{1}$, Annie Arvidsson ${ }^{2}$, Thorarinn Gislasson ${ }^{1}$, Jón Snædal ${ }^{3}$, ${ }^{1}$ Landspitali University Hospital, Reykjavik, Iceland; ${ }^{2}$ AstraZeneca, Sodertalje, Sweden; ${ }^{3}$ Memory Clinic, Reykjavik, Iceland.

Contacte-mail: khannesd@landspitali.is

Background: Recent findings suggest that obstructive sleep apnea (OSA; a disorder associated with abnormal pauses in breathing and intermittent chronic hypoxia during sleep) is common in the early stages of dementia and there is a growing interest in studying the cognitive and neuropsychiatric consequences of OSA in Alzheimer's disease (AD).This study aims to explore the prevalence and nightly variance in OSA in mild AD in relation to repeated assessments of cognitive function. This study builds on two recent pilot studies previously presented at AAIC (Hannesdottir et al., 2011 and 2012); one revealing a high frequency (87.5\%) of OSA in 24 mild cognitive impairment (MCI) and mild dementia and the other indicating fluctuations in OSA severity in mild AD. Methods: Twenty mild AD patients (MMSE > 20; age 45-80 years), over a time period of four weeks, underwent five overnight sleep studies including pulse oximetry, assessment of respiratory effort, airflow, body position and movement. Patients were divided into two groups based on their apnea-hypopnea index (AHI); ten patients with an average $\mathrm{AHI}<10$ and ten patients with an average $\mathrm{AHI} \geq 10$. CogState computerized cognitive assessments were performed each morning following the sleep assessments to measure visual memory and attention/ vigilance and working memory. Participants additionally completed questionnaires intended to measure signs of OSA such as physical symptoms, daytime sleepiness and snoring. Results: Initial results revealed an OSA frequency of $90 \%(\mathrm{AHI}>5)$ and a mean $\mathrm{AHI}$ of $13.0(5.7 \mathrm{SD})$ per hour over 5 assessments (66\% had mild OSA, 17\% moderate and 17\% severe). Substantial nightly fluctuations in OSA were seen with an average fluctuation of 13.4 (9.9 SD) AHI events/hour. Moreover, patients with an average $\mathrm{AHI}<10$ performed significantly better on a test of attention and vigilance than patients with an average $\mathrm{AHI} \geq 10$. Conclusions: We propose that OSA may be underdiagnosed in $\mathrm{AD}$ and that repeated assessments may lead to a more accurate diagnosis of OSA. Preliminary findings suggest cognition in AD may be impacted by the severity of OSA. Further studies are warranted to better understand the effects of OSA on cognitive functioning in AD.

\section{P4-363 OBSTRUCTIVE SLEEP APNEA AND NEUROPSYCHIATRIC SYMPTOMS IN MILD ALZHEIMER'S DISEASE}

Solveig Davidsdottir ${ }^{1}$, Arndis Valgardsdottir ${ }^{1}$, Erna Arnardottir ${ }^{1}$, Annie Arvidsson ${ }^{2}$, Thorarinn Gislasson ${ }^{1}$, Jón Snædal ${ }^{3}$,

Kristin Hannesdottir ${ }^{1},{ }^{1}$ Landspitali University Hospital, Reykjavik, Iceland; ${ }^{2}$ AstraZeneca, Sodertalje, Sweden; ${ }^{3}$ Memory Clinic, Reykjavik, Iceland. Contact e-mail: khannesd@landspitali.is

Background: There is growing interest in studying sleep in Alzheimer's disease $(\mathrm{AD})$ as studies increasingly reveal sleep and circadian disorders, such as irregular sleep, daytime sleepiness, nighttime wandering, sun downing and obstructive sleep apnea (OSA), to be important features of the illness. Research on the relationship between sleep disorders and neuropsychiatric symptomatology in $\mathrm{AD}$ is currently lacking. Recent findings suggest that OSA may be highly underdiagnosed in the early stages of dementia. OSA is a sleep disorder associated with wide ranging clinical consequences in otherwise healthy adults, including neuropsychiatric symptoms such as anxiety and depression. This study aims to explore the prevalence and severity of OSA in mild AD in relation to neuropsychiatric symptoms and daytime sleepiness. Methods: 20 mild AD patients (MMSE > 20; age 45-80 years), over a time period of four weeks, underwent five overnight sleep studies including pulse oximetry, assessment of respiratory effort, airflow, body position and movement. Patients were divided into two groups based on their average AHI; ten patients with an $\mathrm{AHI}<10$ and ten patients with an $\mathrm{AHI} \geq 10$. The Neuropsychiatric Inventory was 